\title{
Prevalence of human papillomavirus infection of 65,613 women in East China
}

\author{
Yanmei Ge${ }^{1}$, Shanliang Zhong ${ }^{2^{*}}\left(\mathbb{D}\right.$, Meng Ren ${ }^{3}$, Yanping $\mathrm{Ge}^{4}$, Yuan Mao ${ }^{1}$ and Peng Cao ${ }^{1}$
}

\begin{abstract}
Background: The infection with human papillomavirus (HPV) is the major cause of genital disease. This study assessed the prevalence and genotype of HPV among outpatient women and healthy women in Jiangsu, East China.

Methods: A total of 65,613 women aged 16-85 years were recruited from Nanjing Kingmed Diagnostics, including 45,736 outpatients and 19,877 healthy women. The cervico-vaginal cells were collected and then HPV types were detected using the Tellgenplex ${ }^{\mathrm{TM}}$ HPV DNA Test.

Results: The overall HPV prevalence was $17.7 \%$ for outpatients and $10.6 \%$ for healthy women. $13.7 \%$ outpatients were infected with a single HPV type and 4.0\% were infected with multiple HPV types. Regarding healthy women, 8.5 and 2. $1 \%$ were infected with single and multiple HPV types, respectively. The two most commonly detected HPV types were HPV 16 and 58 regardless of single- or multiple-type infection or source of the participants. HPV $16+58$ was the most commonly identified multiple genotype in outpatients, while HPV16 + 52 was frequently detected in healthy women. Highest prevalence rate was found in outpatients aged $<20$ years and $\geq 60$ years.
\end{abstract}

Conclusions: This study revealed the prevalence characteristics of HPV in both outpatient women and healthy women in Jiangsu province.

Keywords: Human papillomavirus, Prevalence, Cervical cancer, Vaccines

\section{Background}

The infection with human papillomavirus (HPV) is strongly associated with genital disease. Type-specific persistent infection with high-risk (HR) HPV is the major reason of cervical squamous intraepithelial lesions as well as cervical cancer [1-3]. According to its relationship with cervical cancer and precancerous lesions, HPV genotypes are divided into HR-HPV and low-risk (LR) HPV genotypes [4]. These different HPV genotypes have different pathogenicity [5]. The HR-HPV genotypes may lead to cervical carcinogenesis, while the LR-HPV are associated with benign or low-grade changes in cervical cells, such as genital warts $[6,7]$.

There are many reports on the prevalence of HPV in different populations. The prevalence of HPV infection varies greatly by country, region within country, and population $[8,9]$. Therefore, understanding the prevalence

\footnotetext{
* Correspondence: slzhong@njmu.edu.cn

${ }^{2}$ Center of Clinical Laboratory Science, Jiangsu Cancer Hospital \& Jiangsu Institute of Cancer Research \& The Affiliated Cancer Hospital of Nanjing Medical University, Baiziting 42, Nanjing 210009, China

Full list of author information is available at the end of the article
}

characteristics of HPV in different regions is very important for the early detection and prevention of local cervical cancer. In the present study, we made a comprehensive analysis of the prevalence characteristics of HPV in both outpatient women and healthy women who were recruited from Jiangsu, China.

\section{Methods}

\section{Study population}

The women who received HPV screening at Nanjing KingMed Diagnostics between January of 2016 and May of 2017 were recruited. We excluded the women who did not have valid testing results (e.g., the unsuccessful test as a result of unqualified sampling) or who were not the residents of Jiangsu Province. Finally, we enrolled 65,613 women aged 16-85 years, including 45,736 outpatients and 19,877 healthy women who received routine examination. All the data were retrospectively collected from Laboratory Information Management System of Nanjing KingMed Diagnostics.

(C) The Author(s). 2019 Open Access This article is distributed under the terms of the Creative Commons Attribution 4.0 International License (http://creativecommons.org/licenses/by/4.0/), which permits unrestricted use, distribution, and reproduction in any medium, provided you give appropriate credit to the original author(s) and the source, provide a link to the Creative Commons license, and indicate if changes were made. The Creative Commons Public Domain Dedication waiver (http://creativecommons.org/publicdomain/zero/1.0/) applies to the data made available in this article, unless otherwise stated. 


\section{Specimen collection}

All the participants were asked to do not douche or use tampons or vaginal creams, deodorants, or medications for 2 days before the test. The participants were not in menstruation period and refrained from sex for $48 \mathrm{~h}$ before the test. The cervico-vaginal cells were collected from the transformation zone of the uterine cervix by a gynecologist or a trained physician using a standard cytobrush, then suspended in a standard transport medium (STM) for the Tellgenplex ${ }^{\text {TM }}$ HPV DNA Test (Tellgen Life Science Co., Shanghai, China) and stored at $4{ }^{\circ} \mathrm{C}$ until analyzed $(24 \mathrm{~h}-72 \mathrm{~h})$ [10].

\section{HPV genotyping}

HPV types were detected using the Tellgenplex ${ }^{\mathrm{Tm}}$ HPV DNA Test (Tellgen Life Science Co. Ltd., Shanghai, China). It could identify the 27 most common HPV genotypes including 14 HR-HPV genotypes (HPV 16, 18, $31,33,35,39,45,51,52,56,58,59,66,68)$ and 13 LR-HPV/undetermined risk (UR-) HPV genotypes (6, 11, $26,40,42,43,44,53,61,81,82,83)$. Briefly, DNA was extracted, then $100 \mathrm{ng}$ HPV DNA was used as template and added into $20 \mu \mathrm{l}$ reaction mixture that includes $10 \mu \mathrm{l}$ PCR premixed solution, $0.8 \mu \mathrm{l}$ Taq DNA Polymerase and $5 \mu \mathrm{l}$ primer mixture.

\section{Statistical analysis}

The SPSS version 19.0 (IBM, Armork, NY, USA) was used to perform all statistical analyses. HPV prevalence rate was estimated by a proportion and summarized as a percentage, with an exact binomial 95\% confidence interval (95\% CI). Prevalence estimates are presented overall and by age ( $<20$ years, $20-29$ years, $30-39$ years, $40-49$ years, $50-59$ years and $\geq 60$ years). The chi-squared $\left(x^{2}\right)$ was used to test for departure from homogeneity in prevalence across all the 6 age groups. The prevalence was estimated separately for outpatients and healthy women. We used data of healthy women to estimate percentage of women who will be protected by the vaccines targeting different HPV types. $p<0.05$ was considered statistically significant.

\section{Results}

The overall prevalence of HPV infection

In the 45,736 outpatient women, 8081 participants (17.7, 95\% CI: 17.3-18.0\%) were infected with at least one
HPV type, including 6253 participants (13.7, 95\% CI: 13.4-14.0\%) infected with a single HPV type and 1828 participants (4.0, 95\% CI: 3.8-4.2\%) infected with multiple HPV types $(P<0.001)$. The HR-HPV prevalence rate (15.7, 95\% CI: $15.3-16.0 \%)$ was higher than the LR/ UR-HPV rate (7.4, 95\% CI: 7.2-7.7\%) (Table 1).

Regarding 19,877 healthy women, $8.5 \%$ (95\% CI: $8.1-$ $8.9 \%)$ were infected with single HPV subtype, whereas 2.1\% (95\% CI: $1.9-2.3 \%)$ were infected with multiple HPV subtypes $(P<0.001)$. HR-HPV and LR/UR-HPV infection was detected in $2002(10.1,95 \%$ CI 9.7-10.5\%) and 611 (3.1, 95\% CI: 2.8-3.3\%) healthy women, respectively (Table 1 ). We estimated percentage of women who will be protected by the vaccines targeting different HR-HPV types. The estimates are presented in Additional file 1: Table S1. We further analyzed the distribution of HPV types in HPV-positive specimens. The top ten HPV types are presented in Table 2. The two most commonly detected HPV types were HPV 16 and 58 regardless of single- or multiple-type infection or source of the participants.

\section{The prevalence of multiple HPV infection}

In the outpatient women, there were 1336 participants infected with dual HPV types and 492 participants infected with three or more HPV types (Table 1). In healthy women, there were 335 participants infected with dual HPV types and 79 participants infected with three or more HPV types (Table 1). Table 3 presents the top ten combinations of HPV types in the participants. The top three most common combinations of the types were HPV $16+58$, HPV $52+58$ and HPV $39+52$ for outpatient women and HPV16+52, HPV52 +58 and HPV39 + 58 for the healthy women (Table 3). The detailed distributions of multiple HPV infection are presented in Additional file 2: Table S2.

\section{The prevalence of HPV infection in different age groups} We analyzed the distribution of positive HPV results in different age groups. Our results showed that the under 20 age group owned the highest rate of HPV infection (36.7, 95\% CI: $32.2-41.2 \%$ ), followed by the over 60 age group (25.6, 95\% CI: 23.3-27.9\%), while the 30-39 age group had the lowest prevalence rate $(17.0,95 \% \mathrm{CI}$ : $16.4-17.6 \%)$ in the outpatient women $(P<0.001)$. In the

Table 1 The prevalence of HPV infection in all the specimens

\begin{tabular}{lllllll}
\hline & & & & & \multicolumn{2}{c}{ Multiple infection (\%) } \\
\cline { 6 - 7 } Participants & Total (\%) & HR-HPV (\%) & LR/UR-HPV (\%) & Single (\%) & Dual & 1336(2.9) \\
\hline Outpatient women & $8081(17.7)$ & $7160(15.7)$ & $3405(7.4)$ & $6253(13.7)$ & $492(1.1)$ \\
Healthy women & $2110(10.6)$ & $2002(10.1)$ & $611(3.1)$ & $1696(8.5)$ & $335(1.7)$ & $79(0.4)$ \\
$P$ & 0 & 0 & 0 & 0 & 0 & 0 \\
\hline
\end{tabular}


Table 2 The prevalence of the top ten frequences of HPV type of the single/multiple infection in the outpatient and healthy women

\begin{tabular}{|c|c|c|c|c|c|c|}
\hline \multirow[t]{2}{*}{ Infection Type } & \multicolumn{3}{|c|}{ Outpatient women } & \multicolumn{3}{|c|}{ Healthy women } \\
\hline & HPV Type & $n$ & Prevalence rate $(95 \% \mathrm{Cl})$ & HPV Type & $\mathrm{n}$ & Prevalence rate $(95 \% \mathrm{Cl})$ \\
\hline \multirow[t]{10}{*}{ Single infection } & HPV16 & 1018 & $12.6(11.9-13.3)$ & HPV58 & 194 & $9.2(8.0-10.4)$ \\
\hline & HPV58 & 702 & $8.7(8.1-9.3)$ & HPV16 & 187 & $8.9(7.6-10.1)$ \\
\hline & HPV52 & 588 & $7.3(6.7-7.8)$ & HPV52 & 146 & $6.9(5.8-8.0)$ \\
\hline & HPV53 & 338 & $4.2(3.7-4.6)$ & HPV53 & 122 & $5.8(4.8-6.8)$ \\
\hline & HPV39 & 321 & $4.0(3.5-4.4)$ & HPV39 & 105 & $5.0(4.0-5.9)$ \\
\hline & HPV18 & 285 & $3.5(3.1-3.9)$ & HPV61 & 105 & $5.0(4.0-5.9)$ \\
\hline & HPV61 & 278 & $3.4(3.0-3.8)$ & HPV56 & 86 & $4.1(3.2-4.9)$ \\
\hline & HPV56 & 256 & $3.2(2.8-3.5)$ & HPV81 & 82 & $3.9(3.1-4.7)$ \\
\hline & HPV81 & 247 & $3.1(2.7-3.4)$ & HPV59 & 74 & $3.5(2.7-4.3)$ \\
\hline & HPV51 & 227 & $2.8(2.4-3.2)$ & HPV51 & 73 & $3.5(2.7-4.2)$ \\
\hline \multirow[t]{10}{*}{ Multiple infection } & HPV16 & 415 & $5.1(4.7-5.6)$ & HPV16 & 98 & $4.6(3.7-5.5)$ \\
\hline & HPV58 & 406 & $5.0(4.5-5.5)$ & HPV58 & 94 & $4.5(3.6-5.3)$ \\
\hline & HPV52 & 363 & $4.5(4.0-4.9)$ & HPV53 & 75 & $3.6(2.8-4.3)$ \\
\hline & HPV53 & 266 & $3.3(2.9-3.7)$ & HPV52 & 65 & $3.1(2.3-3.8)$ \\
\hline & HPV39 & 243 & $3.0(2.6-3.4)$ & HPV39 & 60 & $2.8(2.1-3.6)$ \\
\hline & HPV56 & 207 & $2.6(2.2-2.9)$ & HPV61 & 56 & $2.7(2.0-3.3)$ \\
\hline & HPV61 & 205 & $2.5(2.2-2.9)$ & HPV56 & 48 & $2.3(1.6-2.9)$ \\
\hline & HPV18 & 191 & $2.4(2.0-2.7)$ & HPV66 & 48 & $2.3(1.6-2.9)$ \\
\hline & HPV81 & 191 & $2.4(2.0-2.7)$ & HPV51 & 43 & $2.0(1.4-2.6)$ \\
\hline & HPV51 & 188 & $2.3(2.0-2.7)$ & HPV43 & 42 & $2.0(1.4-2.6)$ \\
\hline
\end{tabular}

healthy women, people aged $50-59$ years had the highest prevalence rate $(12.4,95 \% \mathrm{CI}: 11.1-13.6 \%)$ and the 30 39 age group had the lowest prevalence rate $(9.9,95 \%$ CI: 9.3-10.6\%) $(P<0.001)$ (Table 4).

We further analyzed the prevalence of single and multiple HPV infections in different age groups. In the outpatient women, the peak prevalence for both single and multiple HPV infection was observed in the participants aged $<20$ years. The lowest prevalence of single HPV infection in outpatients was observed in 20-29 age group, while the lowest prevalence of multiple HPV infection was observed in 30-39 age group. However, it was different from those of the healthy women where the highest prevalence of single HPV infection was observed in 50-59 age group and the lowest was in the over 60 age group. Regarding dual HPV infection in healthy women, the highest and lowest prevalence was respectively observed in the over 60 age group and the 30-39 age group (Table 5).

Table 3 The top ten multiple HPV infection mode in participants

\begin{tabular}{|c|c|c|c|c|c|}
\hline \multicolumn{3}{|l|}{ Outpatient women } & \multicolumn{3}{|l|}{ Healthy women } \\
\hline Combination of types & $n$ & Prevalence rate $(95 \% \mathrm{Cl})$ & Combination of types & $n$ & Prevalence rate $(95 \% \mathrm{Cl})$ \\
\hline $16+58$ & 44 & $2.4(1.7-3.1)$ & $16+52$ & 11 & $2.7(1.1-4.2)$ \\
\hline $52+58$ & 33 & $1.8(1.2-2.4)$ & $52+58$ & 11 & $2.7(1.1-4.2)$ \\
\hline $39+52$ & 21 & $1.1(0.7-1.6)$ & $39+58$ & 9 & $2.2(0.8-3.6)$ \\
\hline $52+61$ & 19 & $1.0(0.6-1.5)$ & $53+58$ & 8 & $1.9(0.6-3.3)$ \\
\hline $16+39$ & 19 & $1.0(0.6-1.5)$ & $16+58$ & 8 & $1.9(0.6-3.3)$ \\
\hline $18+58$ & 18 & $1.0(0.5-1.4)$ & $35+53$ & 6 & $1.4(0.3-2.6)$ \\
\hline $16+56$ & 16 & $0.9(0.4-1.3)$ & $16+51$ & 6 & $1.4(0.3-2.6)$ \\
\hline $16+33$ & 15 & $0.8(0.4-1.2)$ & $16+35$ & 5 & $1.2(0.2-2.3)$ \\
\hline $16+53$ & 15 & $0.8(0.4-1.2)$ & $16+66$ & 5 & $1.2(0.2-2.3)$ \\
\hline $33+58$ & 15 & $0.8(0.4-1.2)$ & $58+61$ & 5 & $1.2(0.2-2.3)$ \\
\hline
\end{tabular}


Table 4 Prevalence of HPV infection in different age groups

\begin{tabular}{|c|c|c|c|c|}
\hline \multirow[b]{2}{*}{ Age groups } & \multicolumn{2}{|c|}{ Outpatient Women } & \multicolumn{2}{|l|}{ Healthy Women } \\
\hline & n (postive/total) & Prevalence rate $(95 \% \mathrm{Cl})$ & n (postive/total) & Prevalence rate $(95 \% \mathrm{Cl}$ \\
\hline$<20$ & $164 / 447$ & $36.7(32.2-41.2)$ & $0 / 0$ & 0 \\
\hline $20-29$ & $1733 / 9886$ & $17.5(6.8-18.3)$ & $327 / 3098$ & $10.6(9.5-11.6)$ \\
\hline 30-39 & $2325 / 13671$ & 17.0(16.4-17.6) & $822 / 8293$ & $9.9(9.3-10.6)$ \\
\hline $40-49$ & 2468/12339 & 20.0(19.3-20.7) & $522 / 4819$ & 10.8(10.0-11.7) \\
\hline $50-59$ & $1032 / 5524$ & 18.7(17.7-19.7) & $332 / 2684$ & $12.4(11.1-13.6)$ \\
\hline$\geq 60$ & $359 / 1401$ & 25.6(23.3-27.9) & $107 / 983$ & $10.9(8.9-12.8)$ \\
\hline P & 0 & & 0.01 & \\
\hline
\end{tabular}

\section{Discussion}

Epidemiological studies had shown that persistent cervical HR-HPV infection is correlated with an increased risk of developing a high-grade cervical intraepithelial lesion (e.g., cervical cancer) [11]. Fortunately, the successful development of HPV vaccines would help to prevent the occurrence of cervical cancer. Population-based epidemiological data would assist to develop HPV vaccines for a specific population.

Most of the previous researches on HPV epidemiology were only based on outpatient samples. Since the outpatients usually come to the hospital for some medical problems, the HPV prevalence is different from that of healthy women who participate in normal medical examinations. In our study, both outpatients and healthy participants were recruited from Jiangsu, China. In the 45,736 outpatient women, the prevalence of HPV was $17.7 \%$ and the HR-HPV prevalence was $15.7 \%$. Compared with similar studies in China, the prevalence in our study was higher than that in Beijing (9.9\%) [12] and Yunnan (12.9\%) [13], but lower than that in Fujian (38.3\%) [14] and Zhejiang (20.54\%) [15]. The different HPV prevalence might be explained by different economic conditions, cultural diversity, geographical regions and survey period. Regarding the 19,877 healthy women, the overall HPV prevalence and HR-HPV prevalence was 10.6 and $10.1 \%$, respectively. It was fully explained that more attention should be paid to the healthy women infected with HR-HPV.

The analysis of HPV prevalence showed that the most commonly identified HR-HPV genotypes were HPV 16, $58,52,39$ and 18 among the outpatient women. It was different from the healthy women, where HPV 58, 16, 52, 39 and 56 were the five most commonly detected HR-HPV genotypes. The present study showed that HPV16, 58 and 52 were the top three detected HR-HPV genotypes in the two populations, which was similar to the other studies conducted in China, despite the prevalence varied widely $[13,16,17]$. However, it was different from the result of a meta-analysis which summarized global reports and indicated that HPV16, 18 and 45 were most commonly detected genotypes [18]. With a relatively lower prevalence for HPV 18, the vaccines against HPV16, 58 and 52 should be developed for Chinese women.

In the outpatient women with multiple HPV infection, the most commonly identified combinations of HPV types were HPV $16+58$, HPV52 + 58 and HPV $39+52$. While the top three combinations of types were HPV16 +52 , HPV52 +58 and HPV39 +58 for the healthy women. It was easy to note that these top three multiple-type infections were the different combinations of HPV 16, 39, 52 and 58. We also found that the vaccines targeting HPV 16/39/52/58 will protect more women in East China, when four HPV types were included in vaccines

Table 5 Prevalence of single and multiple HPV infections in different age groups

\begin{tabular}{|c|c|c|c|c|c|c|c|c|}
\hline \multirow{2}{*}{$\begin{array}{l}\text { Age } \\
\text { groups }\end{array}$} & \multicolumn{4}{|c|}{ Outpatient Women } & \multicolumn{4}{|c|}{ Healthy Women } \\
\hline & Total & Single (\%) & Dual (\%) & 3 or more (\%) & Total & Single (\%) & Dual (\%) & 3 or more (\%) \\
\hline$<20$ & 447 & $90(20.1)$ & $42(9.4)$ & $32(7.2)$ & 0 & 0 & 0 & 0 \\
\hline $20-29$ & 9886 & 1267 (12.8) & 330 (3.3) & $136(1.4)$ & 3098 & $248(8.0)$ & $67(2.2)$ & $12(0.4)$ \\
\hline $30-39$ & 13671 & 1859 (13.6) & $368(2.7)$ & $98(0.7)$ & 8293 & $676(8.2)$ & $112(1.4)$ & $34(0.4)$ \\
\hline $40-49$ & 12339 & $1991(16.1)$ & $362(2.9)$ & $115(0.9)$ & 4819 & $429(8.9)$ & $74(1.5)$ & $19(0.4)$ \\
\hline $50-59$ & 5524 & $788(14.3)$ & $173(3.1)$ & $71(1.3)$ & 2684 & $266(9.9)$ & $55(2.0)$ & $11(0.4)$ \\
\hline$\geq 60$ & 1401 & $258(18.4)$ & $61(4.4)$ & $40(2.9)$ & 983 & $77(7.8)$ & $27(2.7)$ & $3(0.3)$ \\
\hline$P$ & & 0 & 0 & 0 & & 0.031 & 0.001 & 0.992 \\
\hline
\end{tabular}


(Additional file 1: Table S1). If three HPV types were included, HPV 16, 52 and 58 were the recommended combination. When two HPV types were included, HPV 16 and 58 were the recommended combination. 4.6, 3.9 and $2.9 \%$ women will be protected by vaccines targeting HPV 16/39/52/58, HPV 16/52/58, and HPV 16/58, respectively. Because the $2 \mathrm{v}, 4 \mathrm{v}$, and $9 \mathrm{v}$ HPV vaccines on the market do not provide protection against type 39 , and the different prevalence of HPV types was observed between women in China and the other countries, it is necessary to develop HPV vaccines specific for Chinese women. The HPV vaccines that covered the four additional HR-HPV types (HPV16/39/52/58) may be more meaningful for a HPV vaccination program in China.

The peak prevalence rate of overall HPV, single HPV and HR-HPV was observed in healthy women aged 5059 years. This was very different from the findings in outpatients of China. Previous studies have reported that HPV prevalence had two peaks in terms of age, while the peak age varied in different studies [19-21]. The two peaks were generally found in younger and menopausal women. Wang et al. found that HR-HPV infection showed higher rates in younger groups and lower rates in middle age groups and the highest prevalence in individuals aged 15-19 years [22]. Tang et al. reported that individuals aged 21-40years had highest HPV prevalence [23]. Our data suggested that the highest HPV prevalence was found in outpatient women at the ages of $<20$ or $\geq 60$ years.

\section{Conclusion}

In the present study, we presented the data on the prevalence characteristics of HPV in both outpatient women and healthy women who were recruited from Jiangsu province, east of China. Such data could guide interventions to control prevalence of HPV. Vaccines against HPV58/16/52/39 should be developed for Chinese women specifically. 50-59 years old women had highest HPV prevalence and should be paid more attention.

\section{Additional files}

Additional file 1: Table S1. The estimated percentage of women who will be protected by the vaccines targeting different HR-HPV types (XLSX $56 \mathrm{~kb}$ )

Additional file 2: Table S2. The all multiple HPV infection mode in participants. (XLSX $29 \mathrm{~kb})$

\section{Abbreviations}

Cl: Confidence interval; HPV: Human papillomavirus; HR: High risk; LR: Low risk; UR: Undetermined risk

\section{Acknowledgments}

We would like to thank Kingmed Diagnostics for providing the data used in this paper.

\section{Funding}

This study was funded by the National Natural Science Foundation of China (grant number 81602551). The funding body played no role in the design of the study or the collection, analysis, and interpretation of the data or in writing the manuscript.

\section{Availability of data and materials}

The data and materials used during the study are available from the corresponding author on reasonable request.

\section{Authors' contributions}

YMG and SLZ carried out the sample collections, laboratory detection and drafted the manuscript. MR and YPG participated in the design of the study and the statistical analysis. YM and PC participated in the design and laboratory detection. All authors read and approved the final manuscript.

\section{Ethics approval and consent to participate}

This study was approved by the Ethics Committee of Nanjing KingMed Diagnostics. The Ethics Committee of Nanjing KingMed Diagnostics concluded that no informed consent was required because the data are anonymized appropriately.

\section{Consent for publication}

Not applicable.

\section{Competing interests}

The authors declare that they have no competing interests.

\section{Publisher's Note}

Springer Nature remains neutral with regard to jurisdictional claims in published maps and institutional affiliations.

\section{Author details}

'Laboratory of gene amplification, Kingmed Diagnostics, Nanjing 210043, China. ${ }^{2}$ Center of Clinical Laboratory Science, Jiangsu Cancer Hospital \& Jiangsu Institute of Cancer Research \& The Affiliated Cancer Hospital of Nanjing Medical University, Baiziting 42, Nanjing 210009, China. ${ }^{3}$ Institute of Tropical Medicine Third Military Medical University, Chongqing 400037, China. ${ }^{4}$ School of Bioligical Science and Food Engineering, Chuzhou University, Chu zhou 239000, China.

Received: 29 May 2018 Accepted: 25 January 2019

Published online: 11 February 2019

\section{References}

1. Zur Hausen H. Papillomaviruses in human cancers. Mol Carcinog. 1988;1(3): 147-50.

2. Burchell AN, Winer RL, De Sanjosé $S$, et al. Epidemiology and transmission daynamics of genital HPV infection. Vaccine. 2006;24(Suppl 3):S52-61.

3. Muñoz N, Bosch FX, De Sanjosé S, et al. Epidemiologic classification of human papillomavirus types associated with cervical cancer. N Engl J Med. 2003;348(6):518-27.

4. Baseman JG, Koutsky LA. The epidemiology of human papillomavirus infections. J Clin Virol 2005, 32: S16-24.

5. Dunne EF, Markwitz LE. Genital human papillomavirus infection. Clin Infect Dis. 2006;43(5):624-9.

6. Bouvard V, Baan R, Straif $K$, et al. A review of human carcinogens-part B: biological agents. Lancet Oncol. 2009;10:321-2.

7. Cogliano V, Baan R, Straif K, et al. Carcinogenicity of human papillomaviruses. Lancet Oncol. 2005;6:204.

8. De Sanjosé S, Diaz M, Castellsague X, Clifford G, Bruni L, Munoz N, et al. Worldwide prevalence and genotype distribution of cervical human papillomavirus DNA in women with normal cytology: a metal-analysis. Lancet Infect Dis. 2007;7(7):453-9.

9. Krul EJ, Van De Vijver MJ, Schuuring E, et al. Human papillomavirus in malignant cervical lesions in Surinam, a high-risk country, compared to the Netherlands, a low-risk country. Int J Gynecol Cancer. 1999;9(3):206-11.

10. Han J, Swan DC, Smith SJ, et al. Simultaneous amplification and identification of 25 huamn papillomavirus types with Templex technology. $J$ Clin Microbiol. 2006;44(11):4157-62. 
11. Huang $M Z$, Li HB, Nie XM, et al. An analysis on the combination expression of HPV L1 capsid protein and p16INK4a in cervical lesions. Diagn Cytopathol. 2010;38(8):573-8.

12. Li C, Wu M, Wang J, et al. A population-based study on the risks of cervical lesion and human papillomavirus infection among women in Beijing, People's republic of China. Cancer Epidemiol Biomark Prev. 2010;19:2655-64.

13. Li Z, Liu F, Cheng S, et al. Prevalence of HPV infection among 28,457 Chinese women in Yunnan Province, Southwest China. Sci Rep. 2016;6:21039.

14. Wu C, Zhu X, Kang Y, et al. Epidemiology of human papilloma virus infection among women in Fujian, China. BMC Public Health. 2017; 18:95.

15. Chen $\mathrm{X}, \mathrm{Xu} \mathrm{H}, \mathrm{Xu}$ W, et al. Prevalence and genotype distribution of human papillomavirus in 961,029 screening tests in southeastern China (Zhejiang Province) between 2011 and 2015. Sci Rep. 2017:7:14813.

16. Hong $\mathrm{H}, \mathrm{He} \mathrm{TF}, \mathrm{Ni} \mathrm{HX}$, et al. Prevalence and genotype distribution of HPV infection among women in Ningbo, China. Int J Gynaecol Obstet. 2015; 131(1):96-9.

17. Xue $H, L i n X, L i T$, et al. Prevalence and genotype distribution of human papillomavirus infection in asymptomatic women in Liaoning province, China. J Med Virol. 2015;87(7):1248-53.

18. Guan P, Howell-Jones R, Li N, et al. Human papillomavirus types in 115,789 HPV-positive women: a meta-analysis from cervial infection to cancer. Int J Cancer. 2012;131:2349-59.

19. Zhao FH, Lin MJ, Chen F, et al. Performance of high-risk human papillomavirus DNA testing as a primary screen for cervical cancer: a pooled analysis of individual patient data from 17 population-based studies from China. Lancet Oncol. 2010;11(12):1160-71.

20. Zhi YF, Cha XX, Li XF, et al. Prevalence and genotype distribution of human papillomavirus in women in the Henan Province. Genet Mol Res. 2015;14(2): 5452-61.

21. Liu XX, Fan XL, Yu YP, et al. Human papillomavirus prevalence and typedistribution among women in Zhejiang Province Southeast China: a crosssectional study. BMC Infect Dis. 2014;14:708.

22. Wang R, Guo XL, Wisman GB, et al. Nationwide prevalence of human papillomavirus infection and viral genotype distribution in 37 cities in China. BMC Infect Dis. 2015;15:257.

23. Tang $Y$, Zheng L, Yang $S$, et al. Epidemiology and genotype distribution of human papillomavirus (HPV) in Southwest China: a cross-sectional five years study in non-vaccinated women. Virol J. 2017;14:84.

Ready to submit your research? Choose BMC and benefit from:

- fast, convenient online submission

- thorough peer review by experienced researchers in your field

- rapid publication on acceptance

- support for research data, including large and complex data types

- gold Open Access which fosters wider collaboration and increased citations

- maximum visibility for your research: over $100 \mathrm{M}$ website views per year

At $\mathrm{BMC}$, research is always in progress.

Learn more biomedcentral.com/submissions 